\title{
Use of lead adjustment formulas for QT dispersion after myocardial infarction
}

\author{
James M Glancy, Clifford J Garratt, Kent L Woods, David P de Bono
}

\begin{abstract}
Objective-To determine whether lead adjustment formulas for correcting QT dispersion measurements are appropriate in patients after myocardial infarction.

Design-Retrospective analysis of QTc dispersion measurements in 461 electrocardiograms (ECGs). Data are presented as uncorrected QTc dispersion "adjusted" for a number of measurable leads and coefficient of variation of QTc intervals for ECGs in which between six and 12 leads had a QT interval that could be measured accurately.

Patients-Patients were drawn from the placebo arm of the second Leicester Intravenous Magnesium Intervention Trial. Some 163 patients who subsequently died and an equal number of known survivors had ECGs recorded on day 2 or 3 of acute myocardial infarction. ECGs were also available in 135 of these patients from at least 1 month postinfarct.
\end{abstract}

Results-The most common lead in which a QT interval measurement was omitted was aVR $(n=176)$, the least common lead was V3 $(n=13)$. The longest QTc interval measured was most usually in lead V4 $(n=72)$ and the shortest in lead V1 $(n=67)$. As the number of measurable leads decreased there was a small, nonsignificant increase in QTc dispersion from 12 lead to eight lead ECGs (mean (SD) $100(35.5) v 109.5(47.9) \mathrm{ms})$. Lead adjusted QTc dispersion (QTc dispersion/square root of the number of measurable leads) showed a large, significant increase when the number of measurable leads decreased from 12 to eight $(28.9$ (10.3) $v 38.7(16 \cdot 1) \mathrm{ms}, \mathbf{P}<0.001)$. A similar trend was seen for coefficient of variation of QTc intervals (standard deviation of QTc intervals/mean QTc interval 64.3 (2.19) v 8.45 (3.94)\%, P < 0.001).

Conclusions-Lead adjustment formulas for QT dispersion are not appropriate in patients with myocardial infarction. Large differences in lead adjusted QTc dispersion are produced, dependent on the number of measurable leads, for very small differences in QTc dispersion. It is recommended that $Q T$ dispersion is pre- sented as unadjusted QT and QTc dispersion, stating the mean (SD) of the number of leads in which a QT interval was measured.

(Br Heart F 1995;74:676-679)

Keywords: QT dispersion; myocardial infarction; lead adjustment

Increased QT dispersion may be a measure of heterogeneity of ventricular repolarisation and has been proposed as a predictor of patients at risk of ventricular dysrhythmias or sudden arrhythmic death. ${ }^{12}$ There are problems with the methodology of QT dispersion measurement. Doubts about the reproducibility of QT dispersion in normal individuals have been raised. ${ }^{3}$ Moreover, the overlap in QT dispersion between patients and controls seen in published studies also questions its role as a useful predictor of arrhythmic events. ${ }^{45}$

A potential confounding factor in QT dispersion measurement is the number of leads in which a QT interval can be accurately measured. The end of the $T$ wave may be difficult to define in one or more leads of abnormal electrocardiograms (ECGs) because of the flattening of the waveform, or a $U$ wave may interrupt the $T$ wave end. Such leads may have to be omitted from QT dispersion analysis for a given ECG. QT dispersion is defined as the maximum minus the minimum QT interval across the surface ECG. ${ }^{6} \mathrm{QT}$ dispersion thus depends on the two extreme values of QT interval measured. In normal individuals, as leads are removed from QT dispersion analysis the mean QT dispersion of that population decreases, depending on the number of leads removed. To adjust for this, the formula QT dispersion divided by the square root of the number of measurable leads was devised by Day et al. ${ }^{7}$ An alternative approach to a lead adjusted formula is a formula based on the standard deviation or coefficient of variation of QT intervals for all measured leads. ${ }^{8}$ As well as potentially allowing for differences in the number of measured leads, this approach may have the advantage of providing information about QT dispersion from the entire ECG.

Hnatkova et $a l^{9}$ have recently observed that the application of different lead adjustment formulas causes a large variation in QT disper\footnotetext{
Groby Road, Leicester

Correspondence to: Dr J M Glancy, Academic Clinical Sciences Wing, Glenfield General Hospital,

Department of

Therapeutics,

J M Glancy

J Garratt

Pharmacology,

Department o

Therapeutics,

University of Leicester, Leicester
} 
Table 1 Leads omitted from $Q T$ interval analysis and contribution of leads to $Q T c$ dispersion measurement

\begin{tabular}{lrrrrrrrrrrrr}
\hline & $a V R$ & $a V L$ & $a V F$ & $I$ & II & III & V1 & $V 2$ & $V 3$ & $V 4$ & $V 5$ & V6 \\
\hline No of omitted leads & 176 & 111 & 63 & 79 & 95 & 63 & 72 & 19 & 13 & 40 & 67 & 109 \\
Longest QTc interval & 15 & 31 & 46 & 24 & 43 & 37 & 20 & 32 & 48 & 72 & 53 & 38 \\
Shortest QTc interval & 29 & 30 & 30 & 38 & 47 & 31 & 67 & 32 & 40 & 30 & 52 & 33 \\
\hline
\end{tabular}

sion estimates obtained from 27 ECGs of healthy individuals. ${ }^{9}$ The present study evaluates QT dispersion in ECGs after myocardial infarction to ascertain whether lead adjustment formulas are appropriate in this population.

\section{Patients and methods}

The population studied was drawn from the placebo arm of the second Leicester Intravenous Magnesium Intervention Trial. ${ }^{10}$ We identified all patients who subsequently died and analysed a standard 12 lead ECG recorded on day 2 or 3 of acute myocardial
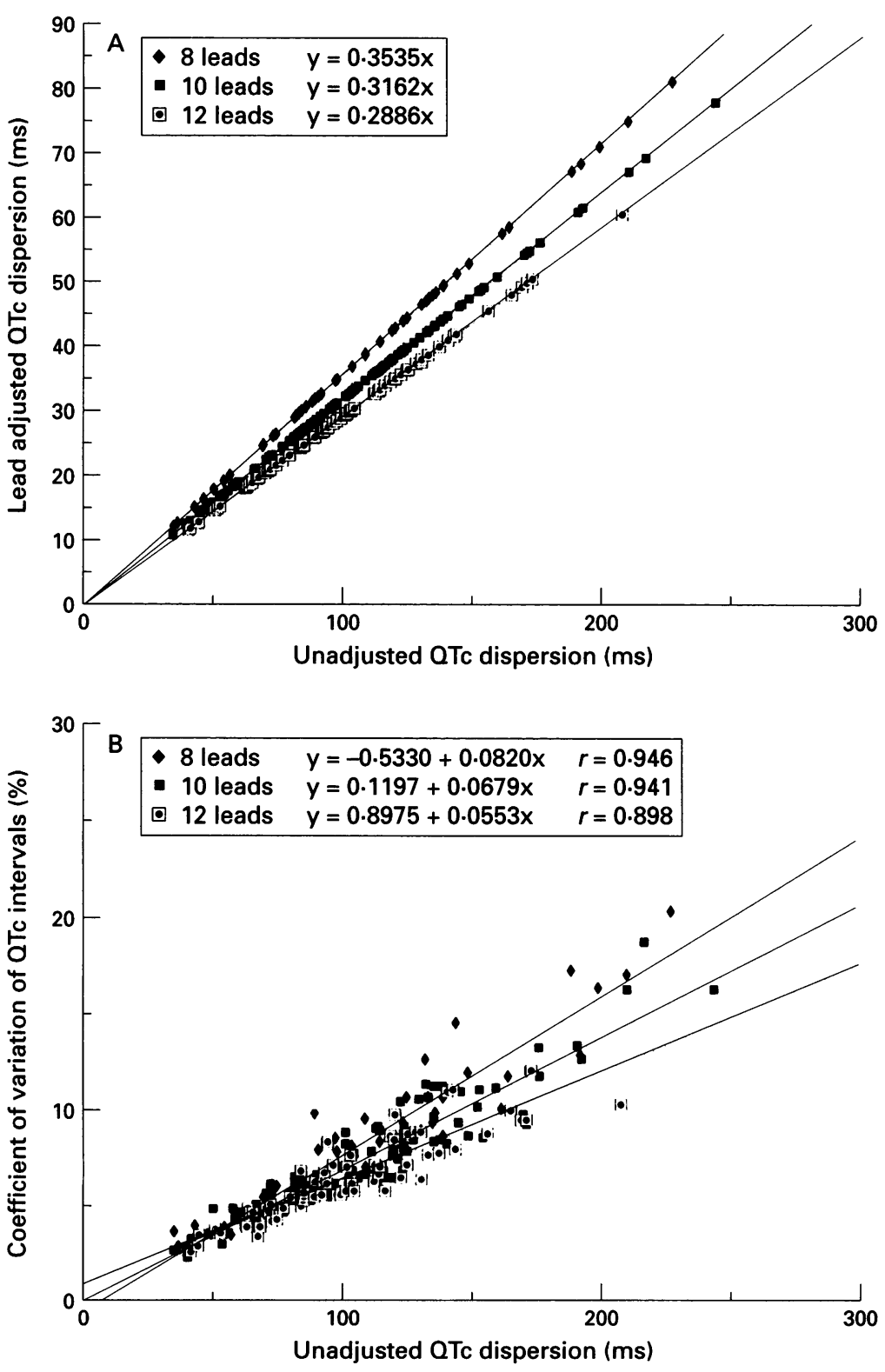

Relation between $(A)$ lead adjusted and unadjusted $Q T c$ dispersion for eight, 10, and 12 leads and $(B)$ between coefficient of variation of $Q T$ intervals and unadjusted $Q T c$ dispersion for eight, 10, and 12 leads. infarction. ${ }^{11}$ ECGs in atrial fibrillation or complete heart block $(n=13)$ were excluded from analysis. Recordings from 163 patients who subsequently died were studied, along with an equal number of age and sex matched longterm survivors. In addition to the early ECG, 53 patients who subsequently died and 82 long-term survivors had available a late ECG recorded at least 1 month after acute infarction. A total of 461 ECGs were examined.

Each ECG had at least five leads in which a QT interval could be accurately measured. A single observer analysed all recordings. ECGs were scanned by a flatbed scanner interfaced with a personal computer. Each image was then cut and copied into 12 files, corresponding to the 12 leads of the ECG. Specially designed software skeletonised and joined each image. Each image can be magnified on the computer monitor for optimal QT interval measurement using a "mouse". This method has been validated previously. ${ }^{12} \mathrm{QT}$ interval measurements were by standard criteria. ${ }^{13}$ Leads were excluded from QT dispersion analysis if the T wave or its terminal portion, was too flat to determine accurately the $T$ wave end; or if a $U$ wave so interfered with a $T$ wave end that the end of the $T$ wave could not be accurately identified. There were no formal criteria for excluding a lead from measurement, this being decided by simple observation by the investigator. QT intervals were rate corrected using Bazett's formula. QTc dispersion is presented using the different formulas:

(1) QTc dispersion is the difference between the longest and shortest rate corrected QT interval across the surface ECG.

(2) Lead adjusted QTc dispersion is the QTc dispersion divided by the square root of the number of leads in which a QT interval was measured.

(3) Coefficient of variation of QTc intervals is the standard deviation of QTc intervals divided by the mean QTc interval. ${ }^{12}$

Statistical analyses were by correlation coefficients and simple regression for the comparison of lead adjustment formulas against QTc dispersion, and Mann-Whitney tests for comparison of inter-lead measurements.

\section{Results}

Table 1 shows the contribution of individual leads to QTc dispersion measurements. Lead aVR was by far the most commonly omitted lead from QT interval measurement, in contrast to leads V2 or V3 which were rarely omitted. The contribution of individual leads as maximum or minimum QT intervals was more evenly spread, lead V4 was over twice as likely to supply a maximum rather than a minimum QT interval, in contrast to lead V1 which showed the opposite trend.

Table 2 shows QTc dispersion measurements and lead adjustment formulas for ECGs with different numbers of leads in which QT intervals could be measured. There was a small, non-significant increase in unadjusted QTc dispersion from 12 to eight leads, followed by a decrease in QTc dispersion from 
Table 2 QTc dispersion, lead adjusted QTc dispersion, and coefficient of variation of $Q T$ T intervals for electrocardiograms (ECGs) in which between six and 12 leads had a measurable QT interval

\begin{tabular}{|c|c|c|c|c|c|c|c|}
\hline $\begin{array}{l}\text { No of measurable leads } \\
\text { No of ECGs } \\
\text { QTc dispersion (ms) }\end{array}$ & $\begin{array}{l}12 \\
68 \\
100(35 \cdot 5)\end{array}$ & $\begin{array}{l}11 \\
103 \\
105 \cdot 4(46 \cdot 3)\end{array}$ & $\begin{array}{l}10 \\
125 \\
103(40 \cdot 1)\end{array}$ & $\begin{array}{l}9 \\
72 \\
110 \cdot 1(45 \cdot 5)\end{array}$ & $\begin{array}{l}8 \\
50 \\
109 \cdot 5(47 \cdot 9)\end{array}$ & $\begin{array}{l}7 \\
28 \\
89 \cdot 5(39 \cdot 3)\end{array}$ & $\begin{array}{l}6 \\
8 \\
93.5(54)\end{array}$ \\
\hline $\begin{array}{l}\text { Lead adjusted QTc } \\
\text { dispersion (ms) }\end{array}$ & $28.9(10.3)$ & $32 \cdot 2(13 \cdot 8)$ & $32.6(12.7)$ & $36 \cdot 6(16)$ & $38 \cdot 7(16 \cdot 1)$ & $33.8(14.9)$ & $38 \cdot 2(22 \cdot 4)$ \\
\hline $\begin{array}{l}\text { Coefficient of variation of } \\
\text { QTc intervals (\%) }\end{array}$ & $6.43(2 \cdot 19)$ & $7 \cdot 15(2.92)$ & $7 \cdot 12(2 \cdot 9)$ & $7 \cdot 84(3.22)$ & $8.45(3.94)$ & $7 \cdot 29(3 \cdot 12)$ & $8.01(4.76)$ \\
\hline
\end{tabular}

Data are mean (SD).

eight to seven leads. There were differences in unadjusted QTc dispersion measurements $(P<0.05)$ between ECGs where seven leads could be measured, and where eight, nine, or 11 leads were analysed. The number of ECGs in which only seven leads had a measurable QT interval was small, however, and for six leads very small. There were large, significant increases in lead adjusted QTc dispersion and coefficient of variation of QTc intervals from 12 to eight leads, and a decrease from eight to seven leads, which reached significance only for lead adjusted QTc dispersion. For lead adjusted QTc dispersion the differences measured between ECGs with different numbers of measurable QT intervals reaches significance at $\mathrm{P}<0.001$ for $12 v$ eight leads; $\mathrm{P}<0.01$ for $12 v 10$ and nine leads, eight $v 10$ and 11 leads; and $P<0.05$ for seven $v$ eight leads. For coefficient of variation of QTc intervals similar results were seen at $P<0.01$ for $12 v$ nine and eight leads; and $P<0.05$ for eight $v 10$ and 11 leads. The figure shows the relation of lead adjusted QTc dispersion and coefficient of variation of QTc intervals with unadjusted QTc dispersion for ECGs in which QT intervals were measured in eight, 10 , and 12 leads.

\section{Discussion}

When measuring QTc dispersion from a 12 lead ECG failure to measure the QT interval in all available leads increases the likelihood that a lead containing a maximum or minimum QT interval will be omitted and tends to lead to an apparent reduction in the measured QTc dispersion. Day et $a l^{7}$ showed that this effect could largely be compensated for by dividing the QT dispersion by the square root of the number of measurable leads.

Application of a valid correction formula to QTc dispersion measurements from a large number of patients would be expected to produce a homogeneous distribution of adjusted QTc dispersion measurements. In fact, while we observed little change in unadjusted QTc dispersion in patients for whom between eight and 12 leads could be measured, the application of the adjustment formula caused a consistent and highly significant increase in "adjusted" QTc dispersion in patients for whom fewer leads could be measured.

One possible explanation is that patients with increased QT dispersion are more likely to have "unusual" ECGs and thus more likely to have one or more leads in which the QT interval is not measurable. An alternative explanation is that in the post-myocardial infarct situation leads for which QTc measurements are most likely to be omitted are in fact those least likely to show either a maximum or minimum QTc value. This would not only explain the apparent overcompensation of the Day formula, but would also explain why coefficient of QTc variation, which in concept should include information from all measured leads, also shows a consistent bias towards a higher value in ECGs for which less than 12 leads have been measured. This hypothesis is difficult to substantiate, as by excluding a lead from measurement it is impossible to know whether it would have included an extreme QT interval.

Lead adjusted QTc dispersion and coefficient of variation of QT intervals may be useful measurements in normal individuals, but are not appropriate in patients after myocardial infarction. Moreover, any simple arithmetical correction factor could be appropriate only if the removal of leads from QT interval measurement in this study was a random process, as would probably be the case in normal ECGs. Our data show that there was a systematic bias in the removal of leads from QT interval measurement.

Our data contrast with the work of Day et al. ${ }^{7}$ In their study of patients treated with sotalol after the acute stage of myocardial infarction QTc dispersion increased in proportion to the square root of the number of leads. They studied a smaller number of ECGs and the population was restricted to patients able to take a $\beta$ blocker. In our study most of the ECGs were recorded on day 2 or 3 of acute infarction. Half of the patients in this study subsequently died and a high proportion had heart failure. Some of the differences in the data between the studies could be based on the difference in the nature of the populations studied.

Small changes in QTc dispersion produce large changes in either adjustment formula, for example, a $9.5 \%$ increase in QTc dispersion from 12 to eight leads is associated with a $33.9 \%$ increase in lead adjusted QTc dispersion and a $31.4 \%$ increase in the coefficient of variation of QT intervals. Rather than adjusting for leads these formulas are producing results biased by the number of leads measured. Simple regression slopes for lead adjusted QTc dispersion are the reciprocal of the square root of the number of leads measured. For different numbers of leads measured in absolute terms greater error is introduced at high values of QTc dispersion, but in relative terms the errors are equal at all values. Similar regression comparing the cor- 
relation of variation of QT intervals to QTc dispersion reveals greater absolute and relative error at high values of QTc dispersion. The regression lines for different numbers of leads crosses at a QTc dispersion of between 50 and $60 \mathrm{~ms}$, however, suggesting that around this value the coefficient of variation of QT intervals may be a very good lead adjustment factor. Hnatkova et $a l^{9}$ have studied the effect of systematically eliminating leads from QTc dispersion analysis in ECGs from healthy individuals. In this population they found that both lead adjusted QTc dispersion and unadjusted standard deviation of QTc intervals were stable methods of lead adjustment and removed the bias introduced by omitting leads. Their major finding was that the coefficients of variance, of any method of QTc dispersion analysis employed, for incomplete ECGs were surprisingly large compared with those for ECGs in which all 12 leads were measured, and they became greater as more leads were removed. They concluded that although on average lead adjustment formulas may work for a population of normal ECGs, they would not be successful in an individual example.

A potential limitation of the data presented is that all measurements were by a single observer, resulting in possible systematic bias. However, the majority of studies of QT dispersion have been by a single observer, blinded to other data about the studies. We do not believe that the observer could have anticipated the result obtained, and in that sense was "blinded". If the observer here encountered a problem with lead adjustment formulas, then we feel it likely that a similar conclusion would be reached by others.

The data presented suggest that neither lead adjustment formulas based on the square root of the number of measurable leads nor the measurement of the coefficient of variation of QT intervals are valid in patients after myocardial infarction. It is recommended that QT dispersion is presented as unadjusted QT and QTc dispersion, stating the mean (SD) of the number of leads in which a QT interval was measured. An alternative approach requiring evaluation would be to measure QT dispersion from a standard set of a small number of leads.

This work was funded by a British Heart Foundation project

We thank HK Bhullar and JC Fothergill, Department of Engineering, University of Leicester, for designing the software for processing the ECGs.

1 Barr CS, Naas A, Freeman M, Lang CC, Struthers AD QT dispersion and sudden unexpected death in chronic QT dispersion and sailure. Lancet 1994;343:327-9.

2 Buja G, Miorelli M, Turrini P, Melacini P, Nava P. Comparison of QT dispersion in hypertrophic cardiomyopathy between patients with and without ventricular arrhythmia and sudden death. Am $\mathcal{f}$ Cardiol 1993;72 973-6.

3 Kautzner J, Gang Y, Camm J, Malik M. Short- and longterm reproducability of QT, QTc and QT dispersion measurement in healthy subjects. PACE 1994;17: 928-37.

4 Linker NJ, Colonna P, Kekwick CA, Till J, Camm AJ, Ward DE. Assessment of QT dispersion in symptomatic patients with congenital long QT syndromes. $\mathrm{Am}$ Cardiol 1992;69:634-8.

5 Pye M, Quinn AC, Cobbe SM. QT interval dispersion: a non-invasive marker of susceptibility to arrhythmia in patients with sustained ventricular arrhythmias? Br Heart $\mathcal{f}$ 1993;71:511-4.

6 Higham PD, Campbell RWF. QT dispersion. Br Heart $f$ 1994;71:508-10.

7 Day CP, McComb J, Matthews J, Campbell RWF. Reduction in QT dispersion by sotalol following myocardial tion in QT dispersion by sotalol fo
infarcion. Eur Heart $¥ 1991 ; 12: 423-7$.

8 Priori SG, Napolitano C, Diehl L, Schwartz PJ. Dispersion of the QT interval-a marker of therapeutic efficacy in the idiopathic long QT syndrome. Circulation 1994;89: 1681-9.

9 Hnatkova K, Malik M, Kautzner J, Gang Y, Camm AJ. Adjustment of QT dispersion assessed from 12 lead electrocardiograms for different numbers of analysed electrocardiographic leads: comparison of stability of different methods. Br Heart $\mathcal{f} 1994 ; 72: 390-6$.

10 Woods KL, Fletcher S, Roffe C, Haider Y. Intravenous magnesium sulphage in suspected acute myocardial Magnesium Intervention Trial (LIMIT-2). Lancet 1992; 339:1553-8.

11 Glancy JM, Garratt CJ, Woods KL, de Bono DP. OT dispersion and mortality after myocardial infarction. Lancet 1995;345:945-8.

12 Bhullar HK, Fothergill JC, Goddard WP, de Bono DP. Automated measurement of QT interval dispersion from hard-copy ECGs. $\mathcal{f}$ Electrocardiol 1993;26:321-31.

13 Lepeschkin E, Surawicz B. The measurement of the QT interval of the electrocardiogram. Circulation 1952;6 378-88. 